\title{
Avaliação da durabilidade de estruturas de concreto expostas em ambientes industriais do polo petroquímico de Triunfo/RS - Estudo de caso.
}

\author{
Evaluation Of Exposed Concrete Structure In Industrial Environments In Triunfo/RS \\ Petrochemical Complex - Case Study
}
F. Coutinho ${ }^{1}$, F. Pacheco ${ }^{2 *}$, G. Bolezina ${ }^{3}$, G. C. Menegussi ${ }^{4}$, R. Christ ${ }^{5}$, H. Z. Ehrenbring ${ }^{6}$, B. F. Tutikian $^{7}$
*Contato do autor: fernandapache@unisinos.br

\begin{abstract}
${ }^{1}$ Engenheiro Civil, Pós-Graduando, Universidade do Rio dos Sinos - UNISINOS, São Leopoldo, Brasil
${ }^{2}$ Professora Doutora, itt Performance, Universidade do Rio dos Sinos - UNISINOS, São Leopoldo, Brasil

${ }^{3}$ Graduanda em Engenharia Civil, Universidade do Rio dos Sinos - UNISINOS, São Leopoldo, Brasil

${ }^{4}$ Graduanda em Engenharia Civil, Universidade do Rio dos Sinos - UNISINOS, São Leopoldo, Brasil

${ }^{5}$ Professor Doutor, itt Performance, Universidade do Rio dos Sinos - UNISINOS, São Leopoldo, Brasil

${ }^{6}$ Doutor em Engenharia Civil, itt Performance, Universidade do Rio dos Sinos - UNISINOS, São Leopoldo, Brasil

${ }^{7}$ Professor Doutor, Programa de Pós-Graduação em Engenharia civil - PPGEC, itt Performance, Universidade do

Rio dos Sinos - UNISINOS, São Leopoldo, Brasil
\end{abstract}

\begin{abstract}
RESUMO
A agressividade ambiental alusiva aos ambientes industriais se caracteriza como forte (classe III) e muito forte (classe IV), conforme a norma NBR 6118:2014. O concreto, nesse local, pode se apresentar suscetível à ação de ácidos, gases, chuvas ácidas, dentre outras ações que, por sua vez, o afetam quimicamente, impactando em sua durabilidade. A presente pesquisa analisou casos de estruturas em ambiente industrial através do ensaio colorimétrico de frente de carbonatação e do modelo matemático simplificado por Tuutti, além dos ensaios de análises químicas, espectroscopia de infravermelhos (FTIR) e cromatografia gasosa. A análise ocorreu em duas empresas do ramo Petroquímico. Foram utilizadas duas amostras de cada empresa: para a primeira, um pilar (P1) externo de concreto moldado in loco, de 8 anos, e um pilar (P2) externo de concreto moldado in loco, de 13 anos. Já a segunda empresa, com estruturas pré-fabricadas, contou com amostras de um pilar (P3) e de um sistema de vedação vertical (PV1), com idade de 27 anos. Percebeu-se, nas amostras, cobrimentos reduzidos, e o avanço da frente de carbonatação, com $11 \mathrm{~mm}$ no P1 e no caso do $\mathrm{P} 2$, já despassivando a armadura. Com relação às amostras da empresa $\mathrm{B}$, pode-se verificar que o P3 apresentou uma boa resistência à carbonatação, enquanto que o PV1 apresentou os sinais de carbonatação até os $20 \mathrm{~mm}$. Na análise de FTIR, as amostras apresentaram espectros parecidos, com picos em regiões semelhantes, para ambas empresas. Através da cromatografia gasosa, ficou evidente a influência decorrente da presença de um aditivo plastificante usado em concreto e em elastômeros, assim como de uma substância de natureza parafínica, comumente utilizada em produtos derivados do petróleo e adotadas por empresas do polo petroquímico.
\end{abstract}

Palavras-chave: concreto; durabilidade; indústria; carbonatação.

\section{ABSTRACT}


The environmental aggressiveness referring to industrial environments is characterized as strong (class III) and very strong (class IV), according to the NBR 6118: 2014 standard. The concrete, in this place, can be susceptible to the action of acids, gases, acid rain, among other actions that, in turn, chemically affect the concrete, decreasing its mechanical resistance and its alkalinity. This research analyzed cases of structures in an industrial environment through the colorimetric assay of carbonation front and the mathematical model simplified by Tuutti, in addition to chemical analysis tests, infrared spectroscopy (FTIR) and gas chromatography. The analysis took place in two companies in the Petrochemical industry. Two samples from each company were used: for the first, an external pillar (P1) of molded concrete in loco, 8 years old, and an external pillar (P2) of molded concrete in loco, 13 years old, both absent from inspections and maintenance over time; the second company, on the other hand, was built with precast materials, with the samples derived from a pillar (P3) and a vertical sealing system (PV1), both aged 27 years. It was noticed, in the samples, reduced coverings, by the carbonation front and it was possible to verify that the P1 had an $11 \mathrm{~mm}$ front and, in the case of P2, the carbonation had already reached the sample reinforcement. Regarding the samples from company B, it can be seen that P3 showed good resistance to carbonation, while PV1 showed signs of carbonation up to $20 \mathrm{~mm}$. In the FTIR analysis, the samples showed similar spectra, with peaks in similar regions, for both companies. Through gas chromatography, the influence resulting from the presence of a plasticizer additive used in concrete and elastomers, as well as a substance of paraffinic nature, commonly used in petroleum products and adopted by companies in the petrochemical pole, became evident..

Keywords: concrete; durability; industry; carbonation.

\section{INTRODUÇÃO}

O concreto tornou-se um dos materiais mais consumidos no mundo, em decorrência de suas diversas formas de utilização e vantagens. Projeta-se que seu uso se mantenha ao longo dos anos. (KIRCHHOF, 2010; FILHO; CARMONA, 2013). Com esse crescente consumo do concreto, fazse necessário o estudo de suas propriedades duráveis, visto que elas influenciam na vida útil e no desempenho das estruturas. Segundo Mehta e Monteiro (2014), a durabilidade do concreto pode ser definida como a capacidade de resistir às intempéries, aos ataques químicos ou a tantos outros processos de deterioração, sem que haja comprometimento da sua capacidade de uso quando exposto ao ambiente para o qual foi projetado.

Para Amorim (2010), o concreto está sujeito aos efeitos de um conjunto de agentes agressivos que, por sua vez, são decorrentes das condições climáticas e ambientais em que o elemento estrutural está inserido, podendo ser causadores de danos mecânicos, físicos, químicos ou biológicos. Pacheco (2016) cita que as estruturas localizadas em ambiente urbano são expostas aos inúmeros agentes agressivos, trazendo a necessidade de se avaliar as particularidades do ambiente e de como estas possam se relacionar aos parâmetros especificados para as estruturas de concreto.

Frequentemente, as construções são encontradas com nível de degradação além do estimado por problemas da qualidade e durabilidade decorrentes de manifestações patológicas e falhas de execução. (MOLIN, 2016). Entende-se que a falta de intervenções de manutenção preventiva pode gerar severos problemas para o sistema estrutural como um todo.

Sales et al. (2018) afirmam que a agressividade ambiental é estabelecida pelas situações vinculadas às condições climáticas que especificam a exposição externa e/ou interna derivada da presença, ou não, de substâncias agressivas, bem como da umidade e temperatura do ambiente.

A NBR 6118 (ABNT, 2014) cita que "a agressividade do meio está relacionada às ações físicas e químicas que atuam sobre as estruturas de concreto”. Desta forma, pode-se estabelecer relações 
de parâmetros e fenômenos que, a partir destas, justificam a classificação da agressividade e que esta tem forte correspondência com o local de inserção da edificação, sob a influência da temperatura e umidade pontuais, concentração dos agentes de deterioração das estruturas, entre outros. (PACHECO, 2016).

Para os projetos de estruturas correntes, a NBR 6118 (ABNT, 2014) apresenta quatro classes de agressividade ambiental, sendo avaliadas conforme as condições de exposição da estrutura e de suas partes. As classes são: I, II, III e IV, estando relacionadas ao risco de deterioração desde o insignificante, pequeno, grande até o elevado, respectivamente. Neste sentido, os ambientes industriais estão inseridos nas classes de agressividade III e IV, conforme as orientações da NBR 6118 (ABNT, 2014), visto que a classe IV contempla os ambientes quimicamente agressivos, galvanoplastia, armazéns de fertilizantes, indústrias químicas de um modo geral, entre outros.

As regiões industriais são, por definição, espaços que favorecem para o acréscimo expressivo de risco de contaminação das estruturas, pois são ambientes com notória presença de gases e de cinzas, além da umidade relativa do ar. As misturas de gases podem vir a reagir com a presença de óxido de enxofre, ocasionando a produção de ácidos (neste caso, o sulfúrico) que, ao entrar em contato com a superfície das estruturas, de forma diluída em água da chuva, resultará na sua degradação vale salientar que o concreto possui valores de $\mathrm{pH}$ que variam no intervalo de 4,5 e 2,2. (ISAIA, 2011). Quando comparado ao ambiente rural, o industrial tem uma taxa de aceleração cerca de 60 a 80 vezes maior na degradação das estruturas da edificação. (SALES et al., 2018).

Abordando especialmente a indústria petroquímica, há necessidade de análise se há contaminação pelos seus produtos e processos, considerando seu impacto em termos de ambiente. (MARTINS et al, 2015).

Em decorrência das manifestações patológicas, que podem ser geradas nesses ambientes, a norma estabelece critérios de projeto que visam à durabilidade da estrutura. Exemplificando, pode-se destacar a qualidade do concreto da camada de cobrimento, a classe de concreto e a relação água/cimento. Todas essas características variam conforme a classe de agressividade ambiental NBR 6118 (ABNT, 2014). Tais especificações visam mitigar a ação de mecanismos de deterioração. Por exemplo, uma adequada espessura e qualidade no concreto de cobrimento das armaduras pode garantir sua proteção. (HELENE, 1997).

Quando essa proteção física e química da armadura se torna ineficiente em relação à agressividade do ambiente, surgem manifestações patológicas, como o caso do desplacamento do concreto, fissuração da matriz de concreto, da corrosão das armaduras longitudinais e transversais, etc., podendo ocasionar a deformação excessiva da estrutura. (NAKAMURA, 2018).

Saetta et al. (1995) afirmam que a vida útil de uma estrutura de concreto pode ser prevista por meio de modelos matemáticos, que consideram fatores como: espessura e qualidade do cobrimento do concreto e as condições ambientais do meio no qual está inserida. Para Possan (2010) o uso de modelos matemáticos tornam-se ferramentas potenciais para determinar uma forma adequada de representar o comportamento da vida útil ao longo do tempo, além da vida útil de projeto (VUP), tais como ensaio colorimétrico de frente de carbonatação e a Lei de Tutti.

Cabe destacar que diante de mecanismos químicos de ataques das estruturas, pode ser realizado o seu diagnóstico mediante ensaios químicos, buscando identificar compostos que não fazem parte naturalmente de estruturas de concreto. O ensaio de FTIR, por exemplo, é empregado para detectar compostos orgânicos, por ser um método que permite a identificação de grupos funcionais presentes nos compostos. (OLIVEIRA, 2008). Já a análise de cromatografia de gás é bastante empregada por analisar qualitativamente e quantitativamente compostos orgânicos. Tendo uma vista sua aplicabilidade na indústria de petróleo com o uso mais direto na análise de hidrocarbonetos. (KANAUJIA, 2018). 
Considerando o impacto do ambiente industrial nas estruturas de concreto, o presente estudo analisou a frente de agressão sofrida por elementos estruturais aplicando ensaio colorimétrico, somado a ensaios químicos, como será relatado no próximo item.

\section{PROGRAMA EXPERIMENTAL}

O estudo contou com amostras de duas diferentes empresas. A empresa A atua com logística, sendo responsável pelo transporte e estoque de polímeros. A empresa B é destinada à produção de pneus para veículos diversos. Ambas estão localizadas no Polo Petroquímico de Triunfo/RS, região de relevância nesse segmento da indústria, que conta ainda com seis empresas.

\subsection{Empresa A}

Nesta empresa, os materiais transportados são, em sua maioria, o polipropileno (PP), polietileno (PE) e borrachas sintéticas. Os elementos estruturais escolhidos têm idade de 8 anos (P1) e 13 anos (P2), figuras 1 e 2, respetivamente. A escolha se deu baseada na proximidade com fontes poluidoras, como a circulação de veículos e proximidade com estocagem de produtos químicos.

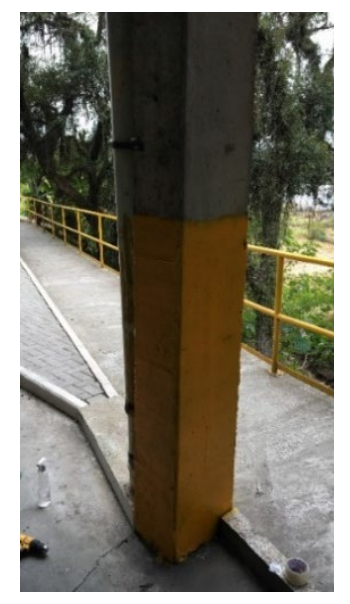

Figura 1. Pilar P1 (empresa A).

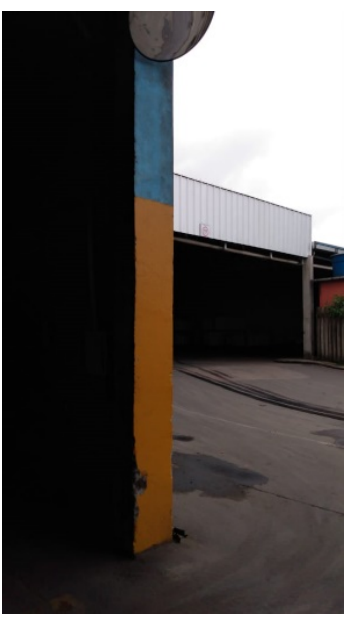

Figura 2. Pilar P2 (empresa A).

\subsection{Empresa B}

A empresa é do segmento de polímeros, atuando com processos de ingestão, transportam a matéria prima em peças automotivas para carros e caminhões como barricas, para-choques, tanques de combustível, dentre outros diversos itens. Os elementos estruturais escolhidos têm 27 anos, ambos (P3) e (PV1), as figuras 3 e 4 apresentam os elementos estruturais, um painel de vedação e um pilar escolhidos para coleta. O painel de vedação encontra-se exposto externamente e o pilar conta com proteção da cobertura. 


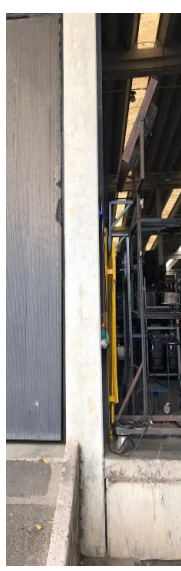

Figura 3. Pilar P3 (empresa B).

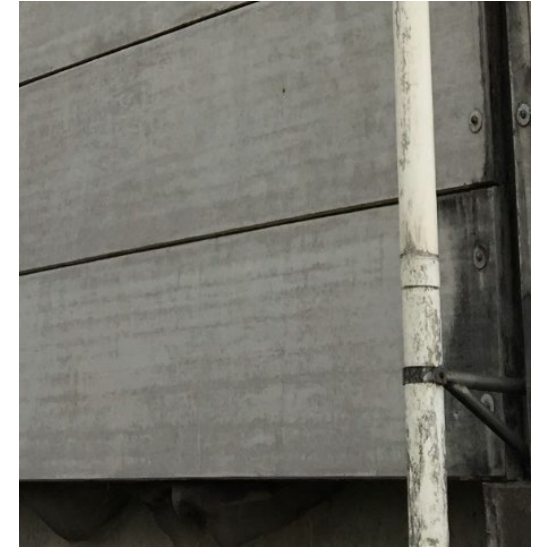

Figura 4. Painel PV1 (empresa B).

\section{MÉTODOS}

\subsection{Visita para vistoria e verificação de manifestação patológicas}

A visita aconteceu nas empresas no mesmo dia. A fim de analisar as manifestações patológicas nas áreas de acesso permitido pelo programa de segurança de cada empresa. A visita serviu para conhecer a edificação e os processos que acontecem, assim como eventuais informações adicionais que auxiliaram na escolha dos pontos de coleta de amostras.

\subsection{Coleta de amostras}

As coletas das amostras e a avaliação das manifestações patológicas seguiram o mesmo para cada uma das amostras, conforme descreve-se:

\subsubsection{Verificação do cobrimento}

Para a aferição do cobrimento da estrutura foi utilizado um detector de partículas eletromagnéticas (da marca Bosch) nos pilares escolhidos. Com o auxílio do equipamento foi obtida a posição da armadura e a possibilidade da localização do mesmo para que não seja atingido nenhuma barra no momento da coleta.

\subsubsection{Limpeza da superfície}

A limpeza ocorreu superficialmente. Inicialmente foi lixada a superfície para remoção do acabamento da área, que foram coletadas as amostras. Após lixar, utilizou-se um pincel.

\subsubsection{Furação e coleta do material}

O processo se deu no uso de uma furadeira com broca de $6 \mathrm{~mm}$ a $1,5 \mathrm{~m}$ de altura, em relação ao chão. $\mathrm{O}$ furo teve a profundidade de até $4,5 \mathrm{~cm}$, atentando-se para não coincidir com a posição das armaduras. Em cada ponto, cuidou-se para que não houvesse variação maior que 1 centímetro da última coleta. Avançou-se na técnica até que houvesse a detecção de concreto são: 

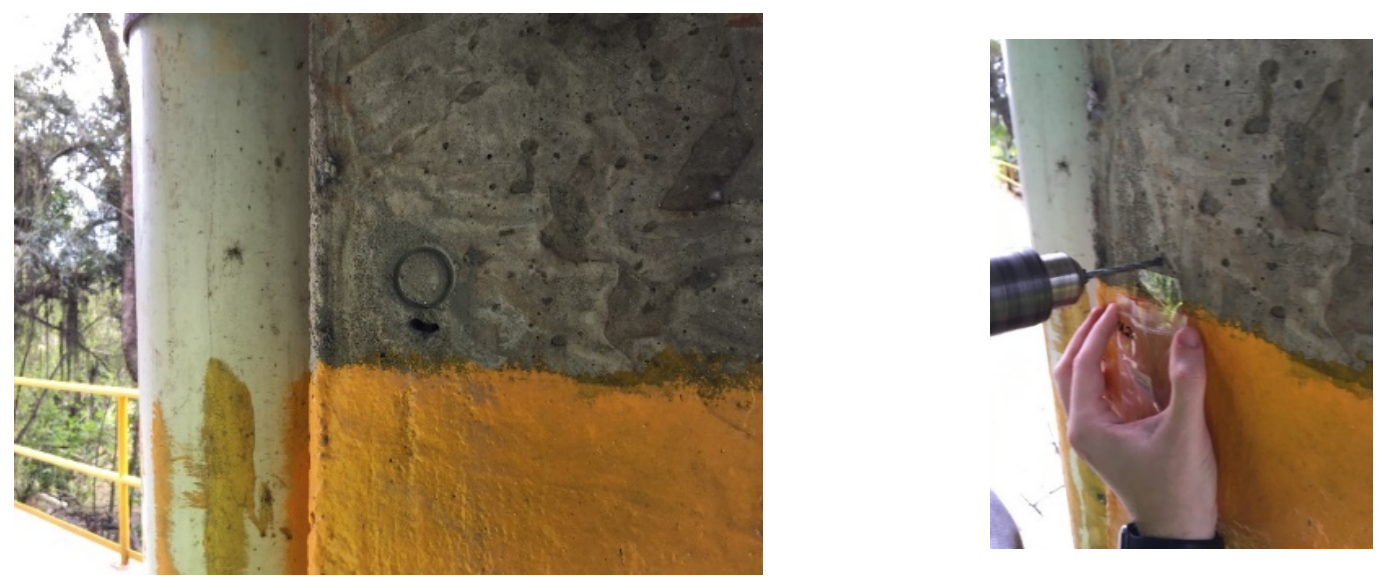

Figura 5. Furos da coleta
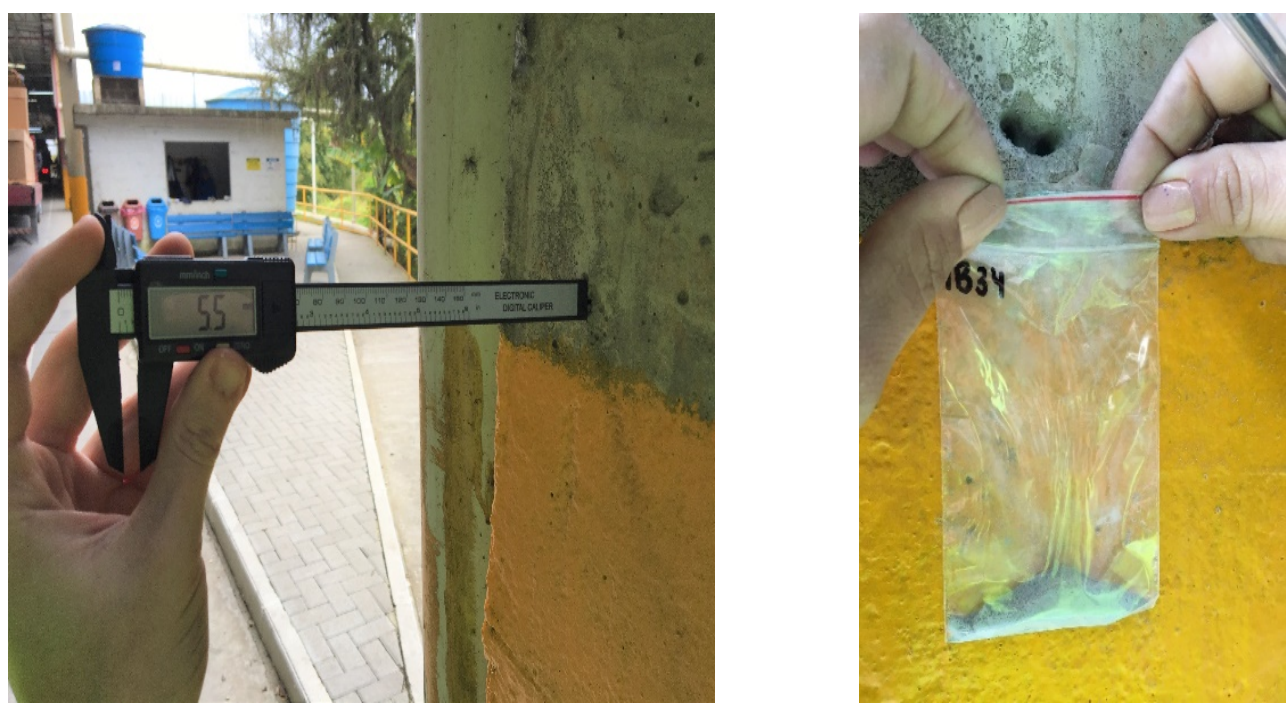

Figura 6. Verificação da profundidade da coleta e armazenamento do pó

\subsection{Ensaio colorimétrico - aspersão de fenolftaleína}

Após as coletas, foi aspergida uma solução à base de $1 \%$ de fenolftaleína, $49 \%$ de álcool etílico e $50 \%$ de água deionizada separadamente nas amostras, como indicador colorimétrico. $\mathrm{O}$ teste feito no local logo após a coleta. Quando o resultado apresenta uma cor carmim é dito que o $\mathrm{pH}$ ainda se mantém alto, se não apresentar alteração, é dito que o mesmo se encontra com pH mais baixo (carbonatado).

\subsection{Aplicação do modelo de previsão de vida útil}

De posse dos resultados obtidos, aplicou-se a previsão de vida útil pelo modelo de Tuutii (1982), seguindo os passos descritos:

1) Verificação da constante $\mathrm{kCO}_{2}: \mathrm{e}=\mathrm{kCO}_{2} \cdot \bigvee_{\mathrm{t}}$

2) Considerando a espessura de cobrimento e a $\mathrm{kCO}_{2}$, obteve-se o tempo em anos;

3) Cálculo da Vida útil residual.

\subsection{Aplicação do modelo de previsão de vida útil}

Os ensaios químicos que foram realizados são cromatografia Gasosa e FTIR (espectroscopia de infravermelho por transformada de Fourier), tendo sido realizados no LBMT (Laboratório de Biologia Molecular e Toxicologia), da Universidade do Vale do Rio dos Sinos - UNISINOS. 


\subsubsection{Cromatografia Gasosa}

Em caráter exploratório, foi realizada análise das possíveis substâncias voláteis e semi-voláteis presentes na composição do concreto. Para esta análise foi realizada uma extração liquido-líquido (LLE) de concreto em solução aquosa com dicloro metano. Em seguida, a extração da amostra foi analisada por cromatografia-gasosa acoplada a espectrometria de massas (GC/MS), para identificar as possíveis substâncias presentes no cimento. Essa identificação foi analisada por comparação/similaridade com o apoio da biblioteca NIST de substâncias químicas. O cromatógrafo gasoso é do modelo 7820A, marca Agilent Technologies, e o espectrômetro de massas é do modelo 5975, marca Agilent Technologies. Para a realização da técnica de cromatografia gasosa é preciso um preparo nas amostras, contando com o seguinte passo-a-passo:

- Pesagem das amostras e separadamente colocadas nos microtubos (eppendorf) de 2ml;

- Adição de $1 \mathrm{ml}$ de diclorometano $\left(\mathrm{CH}_{2} \mathrm{Cl}_{2}\right)$ no eppendorf com a amostra de concreto;

- Uso do vortex durante 30 segundo para agitar a mistura;

- Utilização da centrífuga multispin ao longo de 50 segundos;

- Coleta do sobrenadante com seringa e filtrado com um filtro de 0,45 $\mu \mathrm{m}$;

- Deposição da amostra em recipiente tipo viel;

- Armazenamento em congelador.

\subsubsection{FTIR- Espectroscopia de infravermelho por transformada de Fourier}

Para complementação da caracterização do concreto, as amostras foram submetidas a análise por FTIR, infravermelho por transformada fourier, cujo objetivo é identificar a composição a partir das ligações orgânicas presente na amostra. Essa análise demanda de um preparo simples de redução granulométrica. ${ }^{1}$

\section{RESULTADOS E DISCUSSÕES}

\subsection{Verificação do cobrimento e da vida útil residual 4.1.1 Empresa $A$}

A amostra P1, foi construída no ano de 2011, constataram-se inspeções e manutenções no mesmo. Conforme a NBR 6118 (ABNT, 2014) o elemento estrutural em estudo foi enquadrado como classe IV, com cobrimento mínimo da armadura de $50 \mathrm{~mm}$. O cobrimento aferido por ensaio fora de $37 \mathrm{~mm}$. Para a amostra P2, constatou-se o mesmo enquadramento e cobrimento mínimo. O cobrimento aferido fora de $30 \mathrm{~mm}$. Sendo assim, ambas amostras estão em desacordo em relação ao cobrimento mínimo exigido por norma. Na tabela 1 são apresentados os resultados das amostras P1 e P2, a fim de verificar-se a profundidade de carbonatação, indicando-se na cor magenta a profundidade do concreto são e na cor cinza o concreto já agredido.

Tabela 1. Resultados obtidos na empresa A

\begin{tabular}{|c|c|c|c|}
\hline \multicolumn{4}{|c|}{ EMPRESA A } \\
\hline Elemento & $\begin{array}{c}\text { Cobrimento } \\
\text { aferido (mm) }\end{array}$ & $\begin{array}{c}\text { Profundidade } \\
\text { das amostras } \\
(\mathbf{m m})\end{array}$ & Status \\
\hline P1 - Furo 1 & 37 & 5,5 & \\
\hline P1 - Furo 2 & 37 & 11 & \\
\hline P1 - Furo 3 & 37 & 18,7 & \\
\hline
\end{tabular}

${ }^{1} \mathrm{O}$ equipamento utilizado para a técnica é o FTIR Cary 630, modo ATR, marca Agilent Technologies. 


\begin{tabular}{|l|l|l|l|}
\hline P1 - Furo 4 & 37 & 23 & \\
\hline P2 - Furo 1 & 30 & 5 & \\
\hline P2 - Furo 2 & 30 & 9 & \\
\hline P2 - Furo 3 & 30 & 15 & \\
\hline P2 - Furo 4 & 30 & 26 & \\
\hline P2 - Furo 5 & 30 & 39 & \\
\hline P2 - Furo 6 & 30 & 45 & \\
\hline
\end{tabular}

Foi possível verificar que até os $11 \mathrm{~mm}$, a amostra $\mathrm{P} 1$ apresenta-se carbonatada, apenas entre 18,7 e $23 \mathrm{~mm}$ confirmando a ausência de influência de $\mathrm{CO}_{2}$, que causa o efeito da carbonatação. $\mathrm{Na}$ amostra P2, percebe-se que a carbonatação havia atingido a armadura, devido ao cobrimento da armadura ser inferior a profundidade de carbonatação. $\mathrm{O}$ ensaio foi continuado para verificar o início do ponto de viragem e como percebe-se o mesmo mostrou-se em torno de $45 \mathrm{~mm}$. Para a aplicação do método de Tuutii (1982), considerou-se as informações da tabela 2.

Tabela 2. Dados utilizados para previsão da vida útil

\begin{tabular}{|l|c|c|}
\hline \multicolumn{3}{|c|}{ EMPRESA A } \\
\hline Elemento & P1 & P2 \\
\hline Idade (anos) & 8 & 13 \\
\hline Cobrimento aferido (mm) & 37 & 30 \\
\hline Profundidade de carbonatação adotado (mm) & 23 & 45 \\
\hline
\end{tabular}

$\mathrm{Na}$ amostra $\mathrm{P}$, foi considerada a profundidade de $23 \mathrm{~mm}$, pois não se tem o conhecimento de qual o ponto exato entre 18,7 e $23 \mathrm{~mm}$, considerando assim a condição mais segura de análise.

$$
\begin{gathered}
\mathrm{e}=\mathrm{KCO}_{2} \cdot \sqrt{ }_{\mathrm{t}} \rightarrow 23=\mathrm{KCO}_{2} \cdot \sqrt{ } 8 \rightarrow \mathrm{KCO}_{2}=8,1317 \mathrm{~mm} / \mathrm{ano} \\
\mathrm{e}=\mathrm{KCO}_{2} \cdot \sqrt{\mathrm{t}}_{\mathrm{t}} \rightarrow 37=8,1317 . \sqrt{\mathrm{t}}_{\mathrm{t}} \rightarrow \mathrm{t}=20 \text { anos }
\end{gathered}
$$

Com o resultado do cálculo, é possível afirmar que ainda restam 12 anos para que a armadura seja despassivada, dando início ao processo de corrosão. Segundo Possan (2010), é preciso tomar cuidado com o envelhecimento das estruturas, pois elas alteram suas propriedades mecânicas, físicas e químicas, tanto na superfície como no seu interior, em grande parte dada à agressividade do meio ambiente. Para amostra P2, o método de previsão de vida útil de Tuutti (1982) não se aplicará, pois, a vida útil do mesmo já foi vencida. Tuutii (1982) afirmou que quando a frente de carbonatação atinge a armadura, aceita-se que o período de iniciação a corrosão já foi vencido, pois ela é caracterizada no transporte de gás carbônico para o interior do concreto, podendo desencadear a corrosão.

\subsubsection{Empresa $B$}

A amostra $\mathrm{P} 3$ possui uma idade de 27 anos, durante este período verificou-se que a fábrica não realizou vistorias de manutenções nos elementos estruturais. $\mathrm{O}$ cobrimento da armadura aferido é entorno de 23mm, ao compararmos com a NBR 6118 (ABNT, 2014) há desacordo. A amostra PV1, é um elemento de concreto utilizado para vedação composto por uma malha de ferro. O cobrimento da armadura aferido é entorno de $28 \mathrm{~mm}$. Na tabela 3 são apresentados os dados coletados para as amostras P3 e PV1, a fim de verificar-se a profundidade carbonatação. 
Tabela 3. Resultados obtidos na empresa B

\begin{tabular}{|c|c|c|c|}
\hline \multicolumn{4}{|c|}{ EMPRESA B } \\
\hline Elemento & $\begin{array}{c}\text { Cobrimento } \\
\text { aferido (mm) }\end{array}$ & $\begin{array}{c}\text { Profundidade das } \\
\text { amostras (mm) }\end{array}$ & Status \\
\hline P3 - Furo 1 & 23 & 2,5 & \\
\hline P3 - Furo 2 & 23 & 9 & \\
\hline P3 - Furo 3 & 23 & 12 & \\
\hline PV1 - Furo 1 & 28 & 5,5 & \\
\hline PV1 - Furo 2 & 28 & 11,8 & \\
\hline PV1 - Furo 3 & 28 & 16,2 & \\
\hline PV1 - Furo 4 & 28 & 20,8 & \\
\hline PV1 - Furo 5 & 28 & 26,1 & \\
\hline
\end{tabular}

Foi possível verificar que até os 9mm, a amostra P3 apresentava-se carbonatada, apenas em $12 \mathrm{~mm}$ foi confirmado a ausência da carbonatação. Na amostra PV1, foi possível verificar que até os $16 \mathrm{~mm}$, a amostra apresentava-se carbonatada, apenas entre 20,8 e 26,1 mm foi confirmada a ausência de influência de $\mathrm{CO}_{2}$. Para a aplicação do método de Tuutii (1982), considerou-se as informações da tabela 4.

Tabela 4. Dados utilizados para previsão da vida útil

\begin{tabular}{|l|c|c|}
\hline \multicolumn{3}{|c|}{ EMPRESA B } \\
\hline Elemento & P3 & PV1 \\
\hline Idade (anos) & 27 & 27 \\
\hline Cobrimento aferido (mm) & 23 & 28 \\
\hline $\begin{array}{l}\text { Profundidade de carbonatação } \\
\text { adotado (mm) }\end{array}$ & 15 & 26,1 \\
\hline
\end{tabular}

$\mathrm{Na}$ amostra P3, foi considerado a profundidade de $15 \mathrm{~mm}$ para a aplicação das equações abaixo:

$$
\begin{gathered}
\mathrm{e}=\mathrm{KCO}_{2} \cdot \sqrt{\mathrm{t}} \rightarrow 15=\mathrm{kCO}_{2} \cdot \sqrt{ } 27 \rightarrow \mathrm{kCO}_{2}=2,8868 \mathrm{~mm} / \mathrm{ano} \\
\mathrm{e}=\mathrm{kCO}_{2} \cdot \sqrt{\mathrm{t}} \rightarrow 23=2,8868 . \sqrt{\mathrm{t}} \rightarrow \mathrm{t}=63 \text { anos }
\end{gathered}
$$

Com o resultado, é possível afirmar que ainda restam 36 anos para que a armadura seja despassivada. Para amostra PV1, os resultados das equações, apresentaram que restam 4 anos para que a armadura sofra com a despassivação. Segundo LI et al. (2013), dos principais problemas relacionados a durabilidade das estruturas mostra-se em ambientes com altos níveis de $\mathrm{CO}_{2}$ e/ou íons cloreto, pois, a partir da ação desses agentes há interferência no desempenho, uma vez que eles penetram no concreto e podem causar a deterioração.

\subsection{FTIR}

Os resultados obtidos nas amostras da empresa A, constam nas figuras 7 e 8. As figuras 9 e 10, correspondem aos resultados do ensaio, das amostras P1 e PV1, respectivamente. 


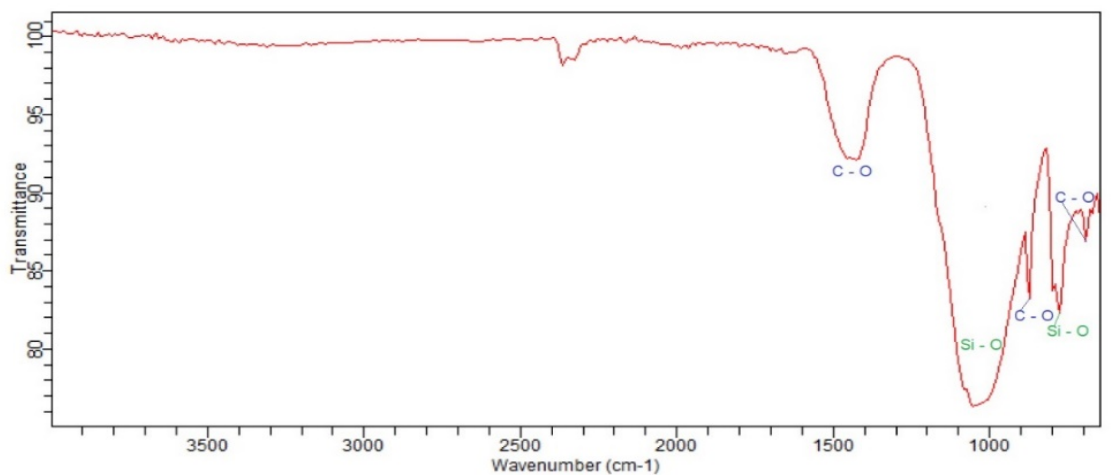

Figura 7. Espectro de infravermelho da amostra P1 (Agilent Technologies (2019)).

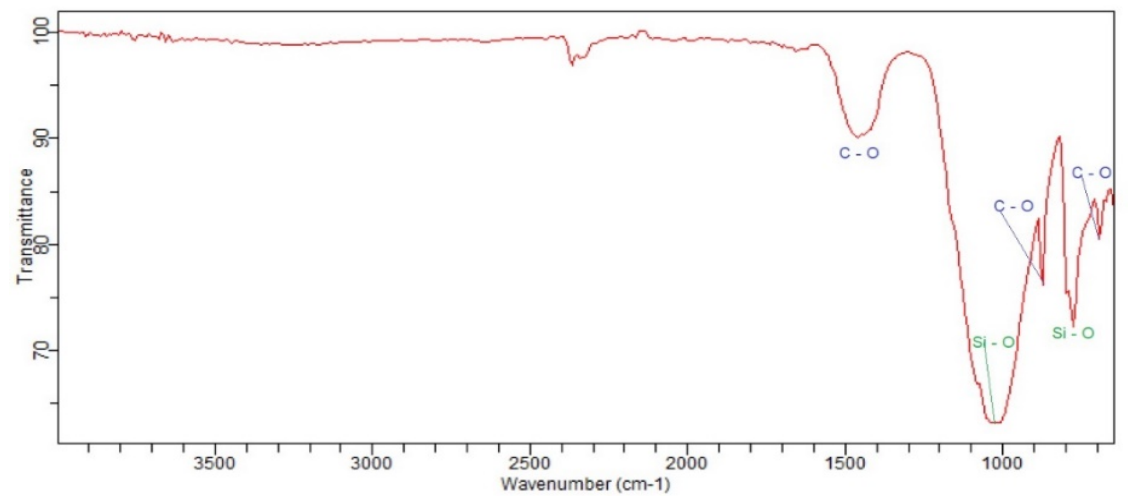

Figura 8. Espectro de infravermelho da amostra P2 (Agilent Technologies (2019)).

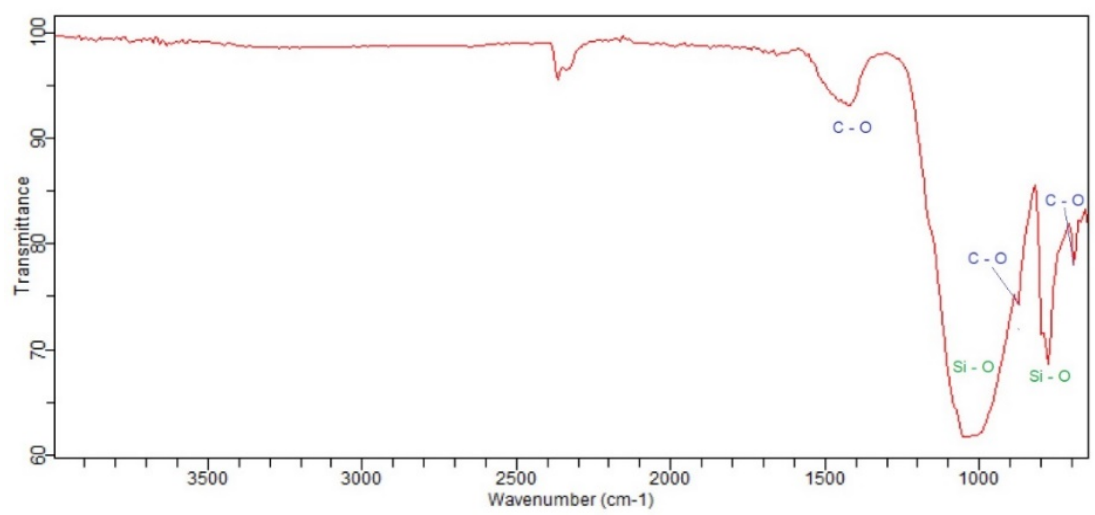

Figura 9. Espectro de infravermelho da amostra P1 (Agilent Technologies (2019)).

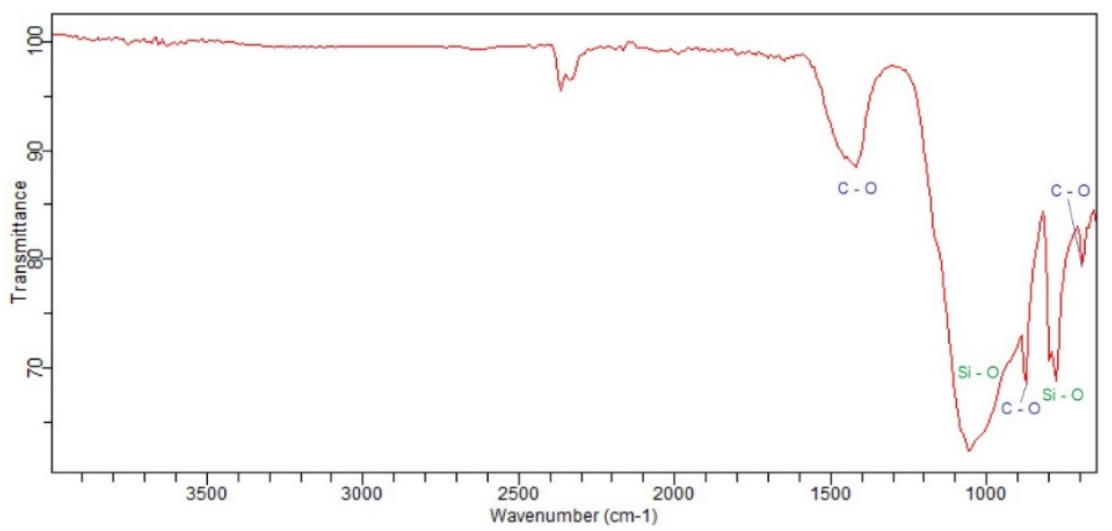

Figura 10. Espectro de infravermelho da amostra PV1 (Agilent Technologies (2019)). 
Na tabela 5 será apresentado o resultado das diferentes bandas do espectro de infravermelho reportadas na literatura para os principais materiais identificados no ensaio.

Tabela 5. Bandas de espectros com identificação

\begin{tabular}{|c|c|c|c|}
\hline \multicolumn{2}{|c|}{ Banda $\left(\mathrm{cm}^{-1}\right)$} & \multicolumn{2}{|c|}{ Identificação } \\
\hline \multicolumn{2}{|c|}{2350} & \multicolumn{2}{|c|}{-} \\
\hline \multirow{4}{*}{$1460-1380$} & 1488 & \multirow{4}{*}{$\mathrm{CO}_{3}{ }^{2-}$ [Andersen 1991] } & aragonita [Andersen 1991] \\
\hline & 1446 & & vaterita [Andersen 1991] \\
\hline & 1445 & & calcita [Andersen 1991 \\
\hline & 1420 & & $\begin{array}{l}\text { dolomita [Reig 2002, Chu 2008, } \\
\text { Rehman 1997, Biscontin 2002] }\end{array}$ \\
\hline \multirow{4}{*}{$1090-1000$} & 1090 & \multirow{2}{*}{$\begin{array}{l}\mathrm{Si}-\mathrm{O} \text { [Reig } 2002 \text { e } \\
\text { Biscontin 2002] }\end{array}$} & \multirow{2}{*}{$\begin{array}{l}\text { quartzo [Reig } 2002 \text { e Biscontin } \\
\text { 2002] }\end{array}$} \\
\hline & 1082 & & \\
\hline & 1034 & \multirow{2}{*}{$\begin{array}{c}\mathrm{Si} \text { - O [Bougeard } 2000 \text { e } \\
\text { Saikia 2003] }\end{array}$} & \multirow{2}{*}{$\begin{array}{l}\text { quartzo [Reig } 2002 \text { e Biscontin } \\
\text { 2002] }\end{array}$} \\
\hline & 1007 & & \\
\hline \multicolumn{2}{|l|}{874} & $\mathrm{CO}_{3}{ }^{2-}[\mathrm{CHU} 2008]$ & calcita [CHU 2008] \\
\hline \multirow{3}{*}{$800-775$} & 798 & \multirow{3}{*}{$\begin{array}{c}\mathrm{Si}-\mathrm{O} \text { [Reig 2002, } \\
\text { Biscontin 2002, Friolo } \\
\text { 2003] }\end{array}$} & $\begin{array}{l}\text { quartzo [Reig 2002, Biscontin } \\
\text { 2002, Friolo 2003] }\end{array}$ \\
\hline & 795 & & caulinita [Friolo 2003] \\
\hline & 779 & & $\begin{array}{l}\text { quartzo [Reig 2002, Biscontin } \\
\text { 2002, Friolo 2003] }\end{array}$ \\
\hline \multicolumn{2}{|l|}{700} & $\mathrm{CO}_{3}{ }^{2-}$ [Andersen 1991] & aragoita [Andersen 1991] \\
\hline
\end{tabular}

A primeira banda em $2350 \mathrm{~cm}-1$ pode-se referir a um ruído ou erro do equipamento. A segunda região de 1460-1380 cm-1 pode corresponder a presença de diferentes variações de carbonato de cálcio, em virtude dos picos nos resultados das amostras se sobreporem. (RODRIGUES, 2013). A dolomita encontrada, segundo Reig (2002), pode ser relacionada a rochas calcárias. A aragonita e a vaterita são formas cristalinas de carbonato de cálcio. (BRANCO, 2008). A Calcita, sendo ela o carbonato de cálcio, tem uma grande importância para rochas de origem carbonática. (RODRIGUES, 2013).

$\mathrm{Na}$ terceira região encontra-se o quartzo, representando o agregado miúdo e a caulinita é classificada como argila mineral, constituída de 66\% de sílica. (GUIMARÃES 2002). Na quarta região também pode-se encontrar a calcita, sendo um produto de alto teor de óxido de cálcio. (GUIMARÃES, 2002). Na quinta região, por sua vez, pode-se encontrar estiramentos de quartzo e caulinita.Na sexta região também há a aragonita.

Segundo Chang e Chen (2004), o ensaio de FTIR pode detectar a carbonatação, os autores afirmam que a presença detectada pela análise pode ser o dobro da detectada usando o indicador de fenolftaleína. A carbonatação é representada pela transformação da $\mathrm{C}=\mathrm{O}$ em $\mathrm{C}-\mathrm{O}$ no $\mathrm{CaCO}_{3}$. Dessa forma, um pico característico do grupo funcional C-O no intervalo de onda de $1410-1510 \mathrm{~cm}-1$ indicaria a carbonatação no concreto. (JCI COMMITTEE 984, 2000). A técnica de FTIR é bastante adequada para monitorar a formação de compostos como carbonatos no concreto, podendo detectar a carbonatação entre 1400-1500cm-1. (YLMÉN; JÄGLID, 2013).

Não foi encontrado água molecular no sistema, sendo caracterizado pela faixa de alongamento $\mathrm{OH}$ em bandas de 3200 a $3700 \mathrm{~cm}-1$, segundo Bhutta et al. (2014). O que poderia indicar que no interior do concreto coletado há resquícios de umidade e pode estar gerando subprodutos, ou até mesmo, dada a idade do elemento, essa água pode estar facilitando a ocorrência da carbonatação. 
Não foi percebido nenhuma outra banda que caracterize, um subproduto formado pela possível degradação do concreto.

\subsubsection{Cromatografia gasosa}

A análise cromatográfica identificou em média 50 compostos de cada amostra, porém foram mantidos apenas os resultados pertinentes do tipo de indústria em estudo. Nas figuras 11, 12, 13 e 14 são apresentados os cromatogramas obtidos oriundos do site Agilent Technologies.

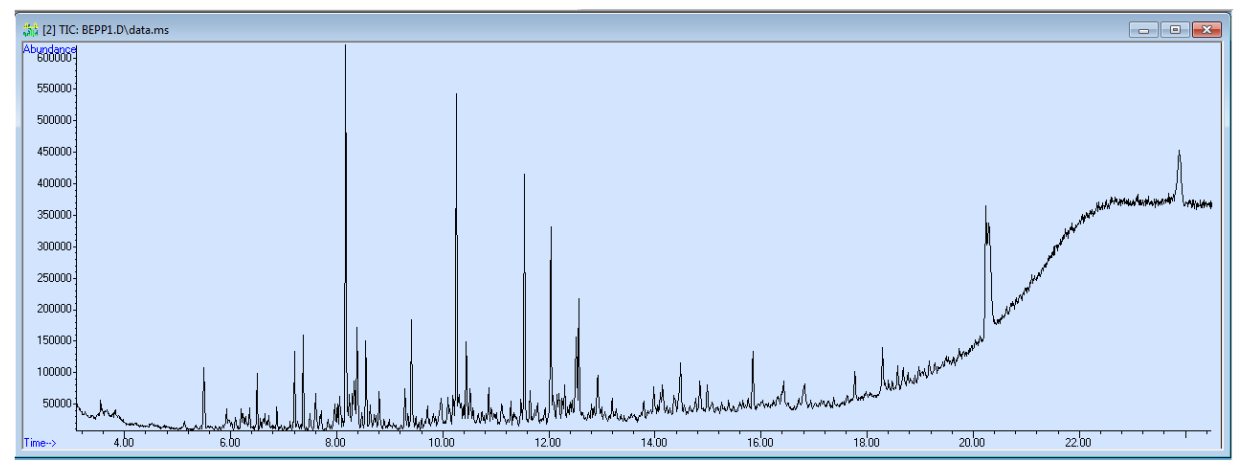

Figura 11. Cromatograma da Empresa A, amostra P1.

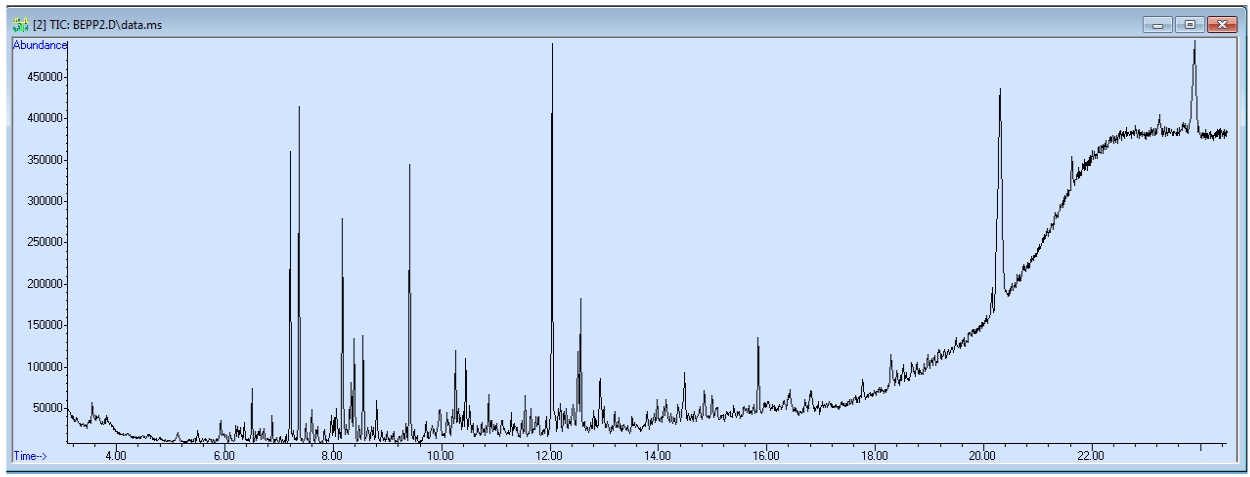

Figura 12. Cromatograma da Empresa A, amostra P2.

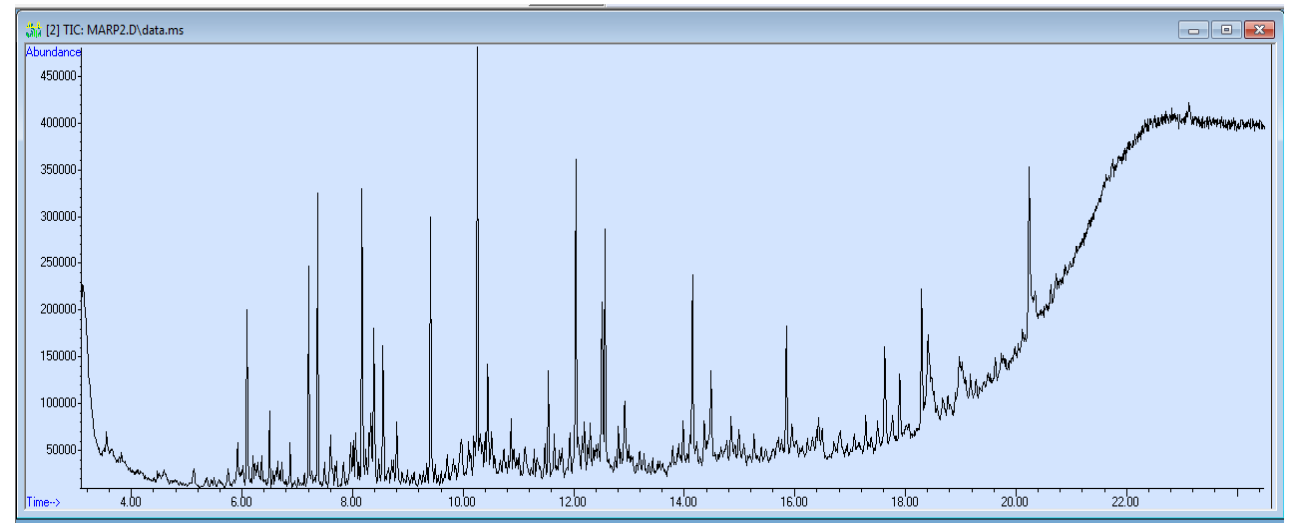

Figura 13. Cromatograma da Empresa A, amostra P1. 


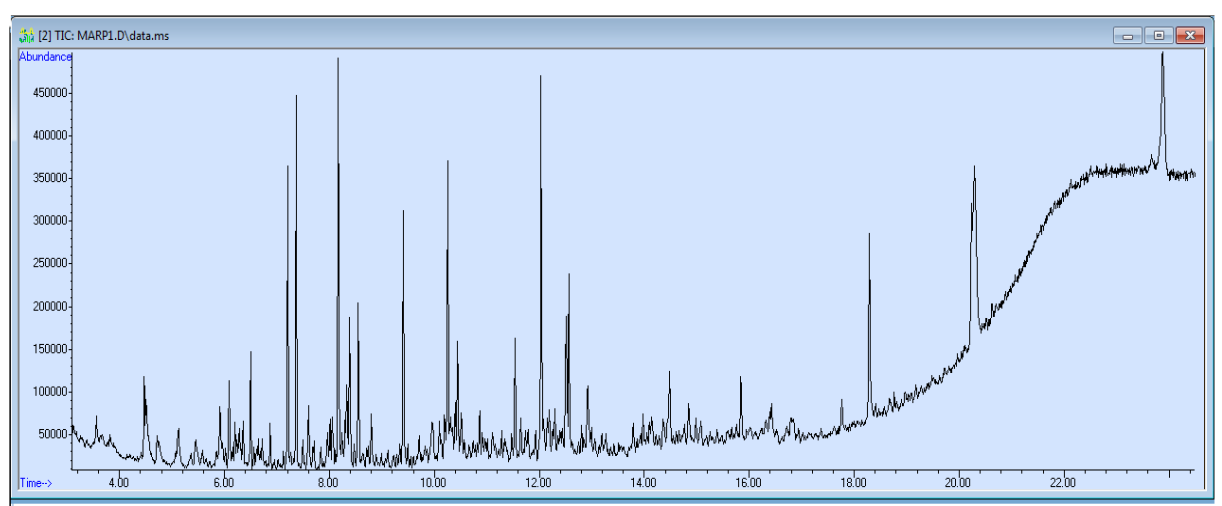

Figura 14. Cromatograma da Empresa B, amostra P1

Tabela 6. Compostos pertinentes ao estudo

\begin{tabular}{|c|c|c|c|}
\hline \multirow{3}{*}{ IDENTIFICAÇÃO } & CAS & $\begin{array}{c}\text { FÓRMULA } \\
\text { MOLECULAR }\end{array}$ \\
\hline \multicolumn{3}{|c|}{ EMPRESA A, P1 } \\
\hline Hexadecane & $000544-76-3$ & $\mathrm{C}_{16} \mathrm{H}_{34}$ \\
\hline Dibutilftalato & $000084-69-5$ & $\mathrm{C}_{16} \mathrm{H}_{22} \mathrm{O}_{4}$ \\
\hline \multicolumn{4}{|c|}{ EMPRESA A, P2 } \\
\hline Dibutilftalato & $000084-69-5$ & $\mathrm{C}_{16} \mathrm{H}_{22} \mathrm{O}_{4}$ \\
\hline \multicolumn{4}{|c|}{ EMPRESA B, P1 } \\
\hline Hexadecane & $000544-76-3$ & $\mathrm{C}_{16} \mathrm{H}_{34}$ \\
\hline Dibutilftalato & $000084-69-5$ & $\mathrm{C}_{16} \mathrm{H}_{22} \mathrm{O}_{4}$ \\
\hline \multicolumn{4}{|c|}{ EMPRESA B, P2 } \\
\hline Hexadecane & $000544-76-3$ & $\mathrm{C}_{16} \mathrm{H}_{34}$ \\
\hline Dibutilftalato & $000084-69-5$ & $\mathrm{C}_{16} \mathrm{H}_{22} \mathrm{O}_{4}$ \\
\hline
\end{tabular}

Segundo Lembo (1999), os hidrocarbonetos cuja formula é $\mathrm{C}_{n} \mathrm{H}_{2 n+2}$ caracterizam-se por parafínicos, assim o composto Hexadecane caracteriza-se como tal. (SILVA, 2005). O petróleo é um óleo mineral constituído por grupos de hidrocarbonetos saturados, sendo eles: (parafinas e naftênicos) (ROCHA et al, 2000); hidrocarbonetos aromáticos; resinas e asfaltenos. (TISSOT; WELTE, 1984). Os produtos petroquímicos são constituídos por etileno, propileno e benzeno. Após o refino do petróleo os produtos mais comuns produzidos são os hidrocarbonetos, sendo eles: liquefeitos; gás de petróleo; gasolina; óleo diesel/ querosene; óleo combustível e parafinas. (LIBARDI, 2009).

O éster dibutilftalato (DBF) é um dos plastificantes mais utilizados na indústria da borracha, ajudando a melhorar as características físicas do produto. Contudo, estudos afirmam que o DBF é um plastificante a base de PVA muito utilizado em concretos, tendo seu principal uso o de aditivo desincorporador de ar. (SILVA, 2007).

O estudo de cromatografia, segundo Jeknavorian et al (2016), mostra que a técnica de cromatografia gasosa pode ser usada tanto para identificar aditivos usados no concreto, quanto para estudar mais a reação do aditivo depois de hidratado o concreto. De acordo com Wexler et al (1963), muitos aditivos podem estar sujeitos a reações de modo a formar outros compostos e 
prejudicar o concreto. Essa análise se mostra interessante, pois os aditivos geralmente têm concentração muita baixa, entre (5 a 5000ppm). (WEXLER et al, 1963).

\section{CONCLUSÃO}

Em relação à empresa $\mathrm{A}$, percebeu-se nos dois elementos avaliados que o cobrimento nominal era inferior ao requerido em norma. Pelo ensaio colorimétrico, um pilar mostrou-se com armadura sã e outro com armadura já despassivada.

Na empresa B, houve desconformidade com a norma, porém, pequena frente de carbonatação, estando os elementos sãos.

$\mathrm{Na}$ análise de FTIR as amostras apresentaram espectros parecidos, com picos em regiões semelhantes. Através da bibliografia foram encontrados minerais normalmente encontrados nas bandas semelhantes. Minerais como aragonita, vaterita, calcita, dolomita, quartzo, caulita, estavam presentes podendo ser precedentes de misturas na fabricação e maturação do clínquer e após do concreto hidratado. A presença de umidade não foi identificada, sabendo que ela apresenta bandas grandes no espectrograma. Com base na literatura a presença de carbonatação pode ser identificada com a técnica de FTIR, o que ocorreu nesse estudo. Não se pode perceber neste ensaio a presença de algum contaminante no concreto ou o que pode ocorrer é que quando há alguma banda com pico grande pode esconder algum pico menos intenso mascarando o resultado.

Através do ensaio de cromatografia gasosa, buscou-se os compostos os que tivessem relação com os possíveis aditivos usados no concreto e suscetíveis residuais do ambiente petroquímico interagindo no concreto. Em todas as empresas foi detectado a possível presença de um composto, o dibutilftato, aditivo plastificante usado tanto nos elastômeros quanto no concreto para melhorar características de ambos, em tal caso não pode afirmar que o mesmo é um residual vindo da indústria petroquímica ou se é comumente utilizado em concreto usinados. Outro composto encontrado na maioria dos elementos analisados foi do Hexadecano, um hidrocarboneto, ele sendo um parafínico é derivado do petróleo, mas não foi encontrado literatura que afirmasse o deu uso em aditivos para concreto ou como contaminante natural.

\section{REFERENCIAS}

AMORIM, E. L. C. de. Ferramentas de Análise de Risco. Apostila do curso de Engenharia Ambiental da Universidade Federal de Alagoas, CTEC, Alagoas: 2010. Disponível em: https://sites.google.com/site/elcaufal/disciplinas/programacao-estruturada. Acesso em: 15 abr. 2020.

ASSOCIAÇÃO BRASILEIRA DE NORMAS TÉCNICAS. ABNT NBR 6118:2014. Projeto de estruturas de concreto — Procedimento. Rio de Janeiro, 2014.

CHANG, J. ., YEIH, W. C., HUANG, R., CHEN, C.T. Suitability of several current used concrete durability indices on evaluating the corrosion hazard for carbonated concrete. Materials Chemistry and Physics, 84(1), 2004, 71-78.

FILHO, C. A., CARMONA, G. T. BT03 - Fissuração nas Estruturas de Concreto. Mérida: ALCONPAT Internacional, 2013. 16 p.

HELENE, P. Vida útil das Estruturas de Concreto In: Congresso Ibere Americano de Patologia das Construções (CONPAT), 4. 1997, Porto alegre. Anais do Congresso Ibwre Americano de Patologia das Construções.

ISAIA, G. C. Concreto: Ciência e Tecnologia. 1. ed. São Paulo: Martins Fontes, 2011.

KIRCHHOF, D. L. Estudo Teórico-Experimental da Influência do Teor de Umidade no fenômeno de Spalling Explosivo em Concretos Expostos a Elevadas Temperaturas. 237 f. Dissertação 
(Doutorado) - Escola de Engenharia, Programa de Pós-Graduação em Engenharia Civil, Universidade Federal do Rio Grande do Sul, Porto Alegre, 2010.

LI, et al. Determination of the apparent activation energy of concrete carbonation. Journal of Wuhan Universaty of Technology-Mater, 2013.

MARTINS, et al. Produção de Petróleo e Impactos Ambientais: Algumas Considerações. Holos, vol 6, 2015, pp54-76 - Instituto Federal de Educação, Ciência e Tecnologia do Rio Grande do Norte.

MEHTA, P. Kumar; MONTEIRO, P. J. M. Concreto: microestrutura, propriedades e materiais. 3. ed. São Paulo: IBRACON, 2014.

NAKAMURA, J. Cobrimento de armaduras determina durabilidade de estruturas de concreto. Disponível em: https://www.aecweb.com.br/revista/materias/cobrimento-de-armadurasdetermina-durabilidade-de-estruturas-de-concreto/14548. Acesso em: 11 abr. 2020.

PACHECO, F. Investigação da Relação Entre os Parâmetros de Projeto das Estruturas de Concreto Armado Visando à Durabilidade. 185 f. Dissertação (Mestrado) - Programa de Pós-Graduação em Engenharia Civil, Universidade do Vale do Rio dos Sinos, São Leopoldo, 2016.

POSSAN, E. Modelagem da Carbonatação e previsão de vida útil de estruturas de concreto em ambiente urbano. 263 f. Dissertação (Doutorado) - Escola de Engenharia, Programa de PósGraduação em Engenharia Civil, Universidade Federal do Rio Grande do Sul, Porto Alegre, 2010. SAETTA, A.V., SCHREFLER, B.A., VITALIANI, R.V., 1995. 2-D model for carbonation and moisture/heat flow in porous materials. Cement Concr. Res. 25 (8), 1703-1712.

SALES, A. et al. Corrosão e Degradação em Estruturas de Concreto: Teoria, Controle e Técnicas de Análise e Intervenção. 2a . Ed. Rio de Janeiro: Elsevier, 2018.

MOLIN, et al. Contribuição à Previsão da Vida Útil de Estruturas de Concreto. In: Avaliação de Desempenho de Tecnologias Construtivas Inovadoras: Materiais e Sustentabilidade. Editora Scienza, 2016.

YLMÉN, R., JÄGLID, U. Carbonation Of Portland Cement Studied by Diffuse Reflection Fourier Transform Infrared Spectroscopy. Int. J. Concr. Struct. Mater. 2013, 7, 119-125. 